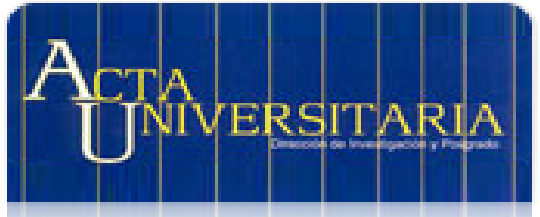

Acta Universitaria

ISSN: 0188-6266

actauniversitaria@ugto.mx

Universidad de Guanajuato

México

Molina Sánchez, Rubén; López Salazar, Alejandra; Contreras Soto, Ricardo

El emprendimiento y crecimiento de las Pymes

Acta Universitaria, vol. 24, núm. 1, diciembre, 2014, pp. 59-72

Universidad de Guanajuato

Guanajuato, México

Disponible en: http://www.redalyc.org/articulo. $0 a ? \mathrm{id}=41648308006$

- Cómo citar el artículo

- Número completo

- Más información del artículo

- Página de la revista en redalyc.org

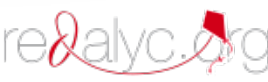

Sistema de Información Científica

Red de Revistas Científicas de América Latina, el Caribe, España y Portugal Proyecto académico sin fines de lucro, desarrollado bajo la iniciativa de acceso abierto 


\title{
El emprendimiento y crecimiento de las Pymes
}

\author{
Entrepreneurship and SME growth
}

Rubén Molina Sánchez ${ }^{*}$, Alejandra López Salazar*, Ricardo Contreras Soto*

\section{RESUMEN}

El presente trabajo pretende sentar las bases para aproximarnos a la postura teórica del emprendimiento regional que explica los diferentes factores de arranque-emprendimiento y el crecimiento de las pequeñas y medianas empresas (Pymes) en las regiones dinámicas. En este trabajo se explica más a detalle las perspectivas que sustentan la teoria, y mostrar a nuestros colegas otra perspectiva de las experiencias acumuladas de nuestras investigaciones en una región de la República mexicana ${ }^{1}$, donde se probaron las premisas de la teoría del emprendimiento.

ABSTRACT

This paper aims to lay the foundation to approach the theoretical position of regional entrepreneurship that explains the different factors start-entrepreneurship and growth of Small and Medium Enterprises (SMEs) in dynamic regions. In this paper we first explain in more detail the perspectives that support the theory and show to our colleagues another standpoint of our research experiences accumulated in a region of Mexico, where the premises of the entrepreneurship theory were tested.

\section{INTRODUCCIÓN}

\section{Antecedentes de la teoría del emprendimiento y crecimiento de las Pymes}

Recibido: 30 de julio de 2013

Aceptado: 31 de julio de 2013

\section{Palabras clave:}

Teoría del emprendimiento; crecimiento de las Pymes.

\section{Keywords:}

Entrepreneurship theory; growth of SMEs.

Cómo citar:

Molina Sánchez, R., López Salazar, A. \& Contreras Soto, R. (2014). El emprendimiento y crecimiento de las Pymes. Acta Universitaria, 24(NE-1), 59-72. doi: 10.15174/au.2014.701 A mediados del siglo pasado se propuso una teoría del crecimiento (Penrose,
1959) en la que se distinguen dos connotaciones del crecimiento. La primera connotación es la tradicional, la que denota un aumento en la empresa, por ejemplo, en ventas, exportaciones, empleos, etcétera; y la otra connotación es la que se refiere a un proceso natural y biológico de crecimiento, que se enfoca en cambios internos que aumentan el objeto. Tradicionalmente, el crecimiento se concibe como algo que de manera gradual sucederá y en donde habrá movimientos hacia un lado u otro, pero no hay nociones de procesos internos de desarrollo.

En suma, esporádicamente se han hecho intentos en la investigación tradicional por desarrollar la teoría del crecimiento de las empresas, usando analogias biológicas y tratando a la empresa como organismos con los mismos procesos de crecimiento que son esenciales para las criaturas vivas del mundo natural (Penrose, 1959).

La teoría del crecimiento se desarrolla primero como una teoría de crecimiento interno; esto es, el crecimiento subyacente detrás de fusiones y adquisiciones (Penrose, 1952). La mayoría de las empresas que sobreviven no

* División de Ciencias Sociales y Administrativas, Campus Celaya-Salvatierra, Universidad de Guanajuato. Ing. Javier Barros Sierra 201, Esq. Av. Baja California, Celaya, Gto., México. C.P. 38140. Tel: (461) 598922, ext. 1602. Correos electrónicos: humanaruben@gmail.com; alelopez.salazar@yahoo.com; riconsoto@hotmail.com 
crecen, y ello es por varias razones: el objetivo inmediato que se encuentra en estas pequeñas empresas es el autoempleo o un trabajo autónomo, lo que limita su crecimiento; esto aunado a una dirección no emprendedora, administración ineficiente, insuficiente capital, aptitud mínima, falta de adaptación a cambios circunstanciales y costosos errores. El talento empresarial es el nuevo elemento de la ecuación que empieza a ser introducido de manera sistemática en la teoría económica, y sus decisiones y acciones son las que constituyen la diferencia entre las empresas que permanecen, las que crecen y las que se estancan o mueren (Suárez-Núñez, 2002). La postura de Penrose (1959) impacta en la reflexión sobre la concepción del crecimiento de la empresa, donde los factores intangibles toman su verdadera dimensión que les corresponde: ver hacia el interior de la organización para potenciar sus recursos y obtener un crecimiento acelerado en la conquista del mercado.

Otra perspectiva donde se sustenta nuestra teoría del emprendimiento es la que se basa en la teoría de los recursos y capacidades de la empresa, teoría que criticó el determinismo de la perspectiva de producto-mercado, y señaló, en cambio, la habilidad de la empresa para influir en el mercado mediante el desarrollo de nuevos productos e innovaciones (Barney, 1986). La perspectiva cambió la atención que tenía en estudiar las acciones e intenciones del competidor, para dar énfasis en la formación y difusión del conocimiento organizacional con el fin de desarrollar las diferencias de la empresa sobre el mercado, como preocupación teórica central. Se propuso localizar las determinantes de la competitividad y crecimiento en las características internas de la organización, porque el comportamiento competitivo no es el resultado del posicionamiento de la organización dentro de un medio ambiente más amplio, sino de ciertas características intrínsecas, únicas, diferentes en cada una de ellas, y de una combinación de recursos únicos e inimitables (Barney, 1986; George, 2005; Hamel \& Prahalad, 1995; Leonard-Barton, 1992; Miles \& Snow, 1984; Prahalad \& Hamel, 1990; Wernerfelt, 1984; 1991).

En contraste con la propuesta de Porter (1982), el enfoque basado en los recursos presta atención a las características internas de la empresa y asume que éstas son heterogéneas en relación con los recursos que poseen, basando en esto sus estrategias, por lo que transferir estos recursos de una empresa a otra es muy dificil (Álvarez, 2003; Barney, 1996; Conner \& Prahalad, 1996;), aunque sí se pueden conjuntar para una alian- za estratégica de colaboración y cooperación interempresarial de un sector determinado como Pymes, donde se unan sus recursos particulares para entrar en mercados mundialmente competitivos.

Como mencionamos anteriormente, encontramos una complementariedad con la perspectiva de las competencias esenciales (Prahalad \& Hamel, 1990) para explicar la ventaja competitiva, buscando el equilibrio en los factores endógenos y exógenos de la empresa. Lo anterior se sustenta en Barney (1986), Conner \& Prahalad (1996), Rumelt (1991) y Wernerfelt (1984) al postular que la perspectiva de los recursos define a la empresa como una colección única $\mathrm{y}-\mathrm{o}$ ante todouna combinación de recursos y capacidades que no se pueden comprar y vender libremente en el mercado. Esto nos obliga a tratar de definir aún más las conceptualizaciones de los recursos en virtud de ser un punto central en la explicación del emprendimiento y crecimiento de las empresas, pero sin considerar otros aspectos exógenos. De ahí que sea necesario que sigamos explorando, después de la conceptualización del término recursos, otras perspectivas teóricas que nos orienten a comprender aún más los factores que determinan el desarrollo de las empresas.

\section{El emprendimiento y crecimiento de la Pyme desde la perspectiva de las competencias esenciales}

En México, en su origen la pequeña industria tuvo como fuente creadora las necesidades primarias del hombre. En este sentido, se formó no sólo como fuente de abastecimiento de bienes para el consumo, sino también fue la activadora del desarrollo de las fuerzas productivas, siendo básicamente la industria de modestos recursos en donde se apoyó la Revolución Industrial y, con ello, la tecnología que hoy en día desarrollan y fomentan las grandes empresas (Rodríguez, 2002). Podemos observar en la figura 1 la aparición de las Pymes, para 1930 la pequeña y mediana industria nacional, por el número de establecimientos, representaba el $12 \%$ del total de la industria de la transformación, en tanto que el restante $88 \%$ correspondia a talleres y artesanías. En 1950, el mismo sector de la pequeña y mediana industria representaba el 25\%, en tanto que los talleres y artesanías se redujeron a $71.9 \%$. En 1955, la pequeña y mediana industria significaba el $45.8 \%$, y los talleres y artesanías el 51.9\%. 
Para 1960, la industria mediana y pequeña representaba el $56.7 \%$, mientras que los talleres y artesanías sólo el 42.8\%. En 1965, la pequeña y mediana industria ascendió a $56.8 \%$, en tanto que la microindustria descendió a 41.8\%. El censo industrial de 1971 reportó un total de 118740 establecimientos de la industria de la transformación, de los cuales $64.4 \%$ correspondian a industrias medianas y pequeñas (Rodríguez, 2002).

Entre 1995 y el primer semestre de 1998, el desarrollo industrial reveló un alto dinamismo de la empresa grande, cuyo número de establecimientos aumentó en $37.2 \%$, que equivalió a 849 nuevas empresas. El número de empresas medianas creció a una tasa de $23.3 \%$ en promedio, lo que significó la apertura de 710 nuevos establecimientos de tamaño mediano, proliferando sobre todo en el segmento de maquiladoras de exportación en el norte del país. En el ámbito de la micro y pequeña empresa, la instalación de 8915 establecimientos estuvo apoyada por la reconversión del mercado. Las pequeñas y medianas empresas poseen características muy convenientes para poder enfrentar la apertura económica internacional en términos de competitividad, no sólo en cuanto a su productividad, sino también en cuanto a su potencial social. Esta clase de empresas ha demostrado en otros países ser un tipo de empresa ideal para conformar el eje de sus economías, como es el caso de Japón, Italia y Brasil.

\footnotetext{
Surgieron 1910 después de la Revolución Méxicana 1930

el $12 \%$ por el número

de establecimientos $y$

$88 \%$ talleres y artesanos
}

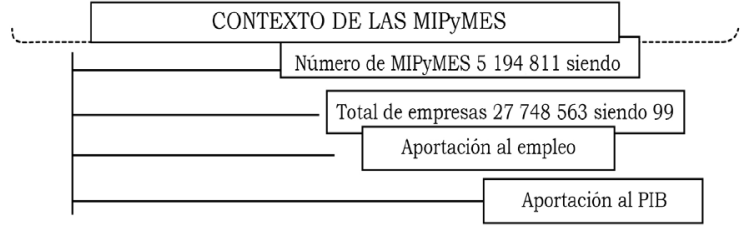

Figura 2. Las micro pequeñas y medianas empresas (MiPymes) y su aportación económica y social, 2009

Fuente: INEGI, 2009.

De acuerdo con los datos de los censos económicos de 2004 y 2009, aparece un incremento de $21.1 \%$ en las unidades económicas, y de $19.6 \%$ en el personal ocupado (Instituto Nacional de Estadística y Geografia [INEGI], 2009). Observaremos en la figura 2 la aportación social y económica en México, siendo en 2009 el 99.9\% de empresas que otorgan a la sociedad el $79.6 \%$ de empleo, y participando económicamente al Producto Interno Bruto (PIB) con el 51.6\%. México se ha abierto al mundo durante las últimas dos décadas: el valor de las exportaciones e importaciones como proporción del PIB aumentó de $38.3 \%$ a $59.6 \%$. Más de tres cuartas partes de las importaciones del país consisten en bienes intermedios, lo que refleja que la mayoría de nuestras compras al exterior se realizan con fines productivos.

Para tener una dimensión de la problemática de mortalidad de las Pymes en México basta mencionar que de cada 100 empresas constituidas, 90 no alcanza a llegar a los dos años de existencia, que es un indice de mortalidad muy elevado, porque en otros países industrializados esta cifra está al menos en $40 \%$.

\section{MATERIALES Y MÉTODOS}

En la presente investigación se utilizó el camino de la investigación cualitativa, de la teoría fundamentada propuesta por Strauss \& Corbin (1998), donde de manera simultánea, como lo plantea Joseph A. Maxwell (1998) citado por González (2006), se dio la acumulación, desarrollo y modificación del marco teórico, a medida que se avanzaba en su construcción. En la investigación cualitativa se utilizó la entrevista como una técnica importante de la etnografia, y permitió la obtención de información que sería muy difícil, si no imposible, conseguir de otro modo, tanto respecto a acontecimientos descritos como sobre perspectivas y estrategias discursivas del entrevistado.

Figura 1. Antecedentes de la aparición de las pequeñas y medianas empresas. Fuente: Datos tomados de Rodríguez, 2002. 
En la investigación se utilizaron tres instrumentos para la recolección de los datos: la entrevista como la principal herramienta para captar la información proporcionada por el propietario-dirigente $(\mathrm{PD})$ de las Pymes, una guía de entrevista flexible y una encuesta con preguntas de opción múltiple. Para diseñar los instrumentos, previa autorización de los autores, se realizó una adaptación de los instrumentos que se utilizaron en el estudio de Julien \& Molina (2012) y el estudio internacional de la Organización para la Cooperación y el Desarrollo Económicos (OCDE) (1998), adicionalmente se incluyen las perspectivas de las competencias esenciales de Hamel \& Prahalad (1995), Prahalad \& Hamel (1990), Zimmerer \& Scarborough (1998), la percepción del PD hacia la innovación de García (1991), Yeung, Ulrich, Nason, Von \& Mary (2000). Asimismo, se agregó la inspección de la tipología del PD de Bruyat \& Julien (2001), Julien \& Marchesnay (1997) y de Miles \& Snow (1984). Finalmente, sobre las necesidades y prácticas de vinculación en red se incluyó el estudio de Julien \& Molina (2012).

Las 48 horas de conversaciones con los PD, que incluyeron las entrevistas, las visitas a las plantas y la aplicación de las encuestas, fueron grabadas y transcritas textualmente en un procesador de textos, en el que resultaron cerca de 50 mil palabras en 80 cuartillas; con esta información se integró la base de datos para ser utilizada por el programa denominado Atlas. Ti vers. 5.1, Scientific Software Development's, 1997; la unidad de análisis fue lo que expresó verbalmente el PD sobre sus experiencias y su contexto en relación con el crecimiento de su empresa. Por cuestiones obvias de espacio, en el presente reporte se describen solamente las matrices elaboradas a partir de las redes semánticas que se generaron en el software Atlas. Ti.

El estudio se delimitó a 12 Pymes manufactureras ubicadas en la ciudad de San Luis Potosí, con la característica de tener de 11 a 50 trabajadores para las pequeñas, y más de 50 para las medianas, además de su experiencia en los últimos diez años como subcontratistas de una empresa grande de clase mundial del área de electrodomésticos, aeronáutica o automotriz, las cuales las señalaron para formar parte de un grupo de 48 Pymes apoyadas económicamente por el Gobierno de este estado, dentro de un programa de asesoría y desarrollo de proveedores con características de crecimiento rápido, en cuanto al incremento del número de empleados o de sus ventas e innovación en procesos y tecnología.
El estudio también se delimitó a 12 casos debido a que el avance del análisis exploratorio mostró que no se requería más información, porque se presentó lo que Glaser \& Strauss (1967) denominan saturación teórica. Estos autores afirman que la saturación teórica se presenta cuando las observaciones adicionales de casos de estudios no conducen a comprensiones adicionales.

\section{RESULTADOS}

En la figura 3 reflexionamos sobre los recursos y capacidades de las Pymes con fuerte crecimiento, respecto a que el emprendedor de aventura tiene el conocimiento claro de las necesidades de su mercado y de los clientes, y tiene identificadas las competencias esenciales soportadas por los recursos y las capacidades al interior de su Pyme. Las Pymes denominadas gacelas deben reorganizarse con frecuencia debido a que si salen adelante es porque se reconfiguran o adaptan con flexibilidad y están bien orientadas al crecimiento.

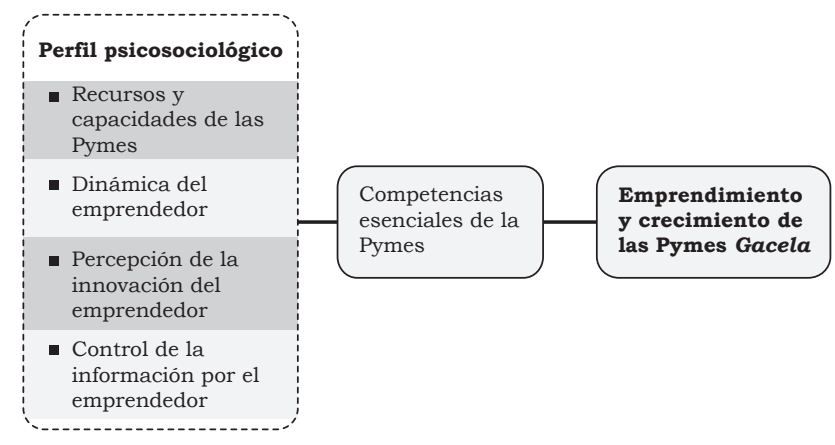

Figura 3. El emprendimiento y el crecimiento de las Pymes desde la perspectiva de las competencias.

Fuente: Adaptado de Julien \& Molina (2012).

\section{Recursos y capacidades de las Pymes}

Wernerfelt (1984) es el primero en publicar un artículo que pondera los recursos basados en la empresa, desde una perspectiva teórica, para recomendar la utilidad de analizar el recurso de las empresas en lugar del producto. El uso de estas herramientas resaltan las nuevas opciones estratégicas que entonces surgen de manera natural de la perspectiva de los recursos de la empresa. Barney (1991) agrega que los recursos son un paquete de ventajas, capacidades, procesos organizacionales, atributos de la empresa, información y conocimiento, controlados por una empresa que la capacitan para 
concebir e implementar estrategias que perfeccionen su eficiencia y eficacia. Asimismo, Barney examina las implicaciones de los recursos de la empresa y la ventaja competitiva sostenida para las diferentes disciplinas del negocio, construyendo el supuesto de que la empresa distribuye de manera heterogénea los recursos estratégicos y que estas diferencias son estables (Barney, 1991; 1996). Grant (1991) identifica cinco importantes categorías de recursos, entendidos éstos desde la conceptualización de Barney (1991): financieros, físicos, humanos, tecnológicos y organizacionales. Los recursos y capacidades de la empresa son considerados claves en la formulación de estrategias, y son la primera constante sobre la cual la empresa es única y estable, además de constituirse en la fuente primaria de la rentabilidad de la empresa. Según Grant (1991), la clave de la investigación basada en los recursos para la formulación de la estrategia es la relación entre capacidades, ventaja competitiva y rentabilidad. Entonces en la economía del conocimiento se demuestra que los recursos se aplican con inteligencia para ofrecer algo diferente a los clientes en una relación particular entre el productor y el consumidor y, por lo tanto, existe una familiaridad particular con las necesidades del cliente y conocimientos especiales para producir una respuesta específica a estas necesidades.

En sintesis, existen esfuerzos por tomar una posición propositiva en los intentos por descubrir los factores determinantes del éxito del emprendimiento y crecimiento de las Pymes, conjuntando las visiones internas y externas, saliéndose de paradigmas rígidos y estáticos, reconociendo la complejidad del fenómeno y adoptando investigaciones más profundas.

\section{Dinámica psicosociológica del emprendedor}

La subcategoría que denominamos dinámica del emprendedor en la figura 3 es útil para explicar, por una parte, la capacidad para la multiplicación y el dinamismo particularmente fuerte de una Pyme y, por otra parte, la red de capital social que tiende al apoyo y soporte de los actores sociales de la Pyme, y que el emprendedor necesita para mejorar sus oportunidades y hacer frente a las dificultades en la consolidación del crecimiento. Un aspecto relevante del emprendedor con los actores sociales es cultivar valores en las relaciones con empleados, clientes y proveedores, como la responsabilidad, bienestar común, lealtad, cooperación, involucramiento, corresponsabilidad, cohesión, confianza, disciplina, trato justo, equitativo y humano, reparto de beneficios, honestidad, reconocimiento, compromiso y seguridad, entre otros. Esto ha sido básico para acceder a diferentes recursos materiales y, antes que nada, inmateriales o de conocimiento, que se representan como dimensiones con gran intensidad dentro de la red de capital social que incluye el aprendizaje y desarrollo de la Pyme promovido por los clientes y proveedores, el reconocimiento de los organismos certificadores en los sistemas ISO, el apoyo de consultores y Gobierno para sus proyectos de crecimiento, y la inclusión en los selectos grupos de subcontratistas de clase mundial de grandes empresas.

Otras características detectadas en las Pymes con fuerte crecimiento es la integración de centros de articulación productiva de Pymes del sector, el soporte institucional público-privado y las relaciones personales fuera del ámbito del negocio. También se observa que a los familiares asociados a la Pyme, en virtud de la dependencia cercana y generacional, les han permitido integrar recursos económicos, técnicos y administrativos para impulsar el crecimiento y, en otros casos, transitar a una independencia de sus familias y, por tanto, la profesionalización interdepartamental para consolidar el crecimiento.

Con respecto a la tipología del emprendedor, en cuanto a actitudes y comportamiento al asumir la conducción de su empresa, fue posible detectar con la investigación el empuje por lograr resultados, la aceptación constante de retos, su habilidad para las relaciones humanas y la persuasión, la acumulación de experiencia y madurez en "el oficio de la empresa", la apertura y flexibilidad para el cambio, una alta tolerancia a la frustración, la persistencia, el apego y la valoración afectiva por el significado de su Pyme, el espíritu emprendedor, la aceptación de riesgos y la cultura del esfuerzo y dedicación. La tipología encontrada está asociada con las actitudes y comportamiento que asume el emprendedor en la conducción de su empresa, y se reflejan por su emprendimiento acertado, destacando en la tipología de valoración por sus rutinas de gestión, fidelidad a sus clientes, valor agregado y estrategia activa, pero, sobre todo, porque procede a cambios cada vez más importantes en la presentación de los servicios que ofrece, adoptando una estrategia más activa. Se observó una gran actividad del emprendedor para vigilar la información de oportunidades de mercado y nuevos nichos en él y la estrategia en los contratos de ventas actuales o potenciales.

Es de destacar la capacidad de comercialización, respaldada por los beneficios que el emprendedor ha conseguido para su empresa y personal, con clientes, proveedores y actores dentro y fuera de la red de su negocio, como el Gobierno y consultores. 


\section{Percepción de la innovación por el emprendedor}

La percepción es desde mediados del siglo pasado tema de interés de la Psicología y recientemente de las Ciencias Sociales y Administrativas, las cuales introducen en sus investigaciones e intervenciones asuntos sobre el cambio y la cultura organizacional, el estudio de la subjetividad e interpretación de la "realidad" como elementos esenciales para entender los diferentes procesos humanos que confluyen en las diferentes organizaciones. En la investigación que citamos encontramos entre los emprendedores evidencias de la percepción de la innovación que predetermina su actitud, al tomar en cuenta la importancia y capacidad de la mente e inteligencia de sus empleados antes que las capacidades de sus máquinas, que la alta tecnología o la sofisticación en los procesos productivos, en algunos casos automatizados; es decir, perciben como lo más importante a la mente de obra en sus trabajadores en lugar de la mano de obra. Esto les permite considerar, en primer término, al elemento humano antes que a la máquina. El emprendedor considera los conocimientos y habilidades de su personal para el encuentro hombre-máquina como el potencial de su Pyme para hacer frente a la operación de los equipos y la tecnología. Esto le permite promover la innovación por la apertura mental hacia ella. Se observa también el acondicionamiento de los equipos de la fábrica y el cambio en los procesos de producción de un producto para reducir los costos y, de esta forma, mejorar la competitividad de la empresa. Igualmente, el diseño especial de la planta en producción, bajo un modelo de flujo tendido y de justo a tiempo, permite responder de forma rápida a las fluctuaciones de la demanda y minimizar los inventarios. Estas pequeñas innovaciones requieren un trabajo en equipo y una gran distribución de las tareas, además de la participación de los empleados en la producción y en la búsqueda de soluciones para los cambios imprevistos. Asimismo, los sistemas de calidad implementados por las Pymes llevan a cada empleado a revisar, en todas las fases de la producción, la calidad de la parte del producto que él controla, y a reaccionar cuando no esté perfecta. Esta innovación sistemática requiere el acompañamiento de acciones particulares, en el plano de la formación de los empleados, ya sea de carácter interno (juntar a un empleado con uno más experimentado, acudir con los consultores para cambiar procesos) o externo, como ofrecer formación específica a determinados empleados.

En la mayoria de las Pymes, la innovación se realiza sin investigación ni desarrollo (I+D) debidamente elaborados; más bien ocurre por pequeños cambios que se hacen en diferentes puntos de la cadena de valor, generando así una innovación global, o bien, que reúne todos esos cambios pequeños, disminuyendo el riesgo de cometer graves errores y de costosos incidentes que afectarian significativamente el crecimiento. Los emprendedores entrevistados tienen la perspectiva de una constante innovación, para darle flexibilidad a sus procesos de producción, cambiar y mejorar continuamente, realizar reflexiones sobre lo que hacen de modo cotidiano, tolerando la improvisación a la vez que establecen el control de las rutinas o procesos, y otorgan un servicio original a sus clientes para obtener ventaja competitiva con la certificación en la calidad de sus procesos y el reconocimiento de sus clientes por sus servicios.

La proximidad con el mercado objetivo de los emprendedores de las Pymes investigadas les permite elaborar los medios adecuados para detectar expectativas latentes o tácitas de sus clientes actuales o potenciales y transformarlas en productos, bienes y servicios con un alto contenido en calidad y precio competitivo. En el análisis tenemos ejemplos de cómo las Pymes protagonizan una vigilancia cuando envían a algunos de sus empleados (o por lo general el emprendedor va directamente) a explorar no sólo las actividades y los proyectos futuros de sus clientes, sino también sus expectativas no satisfechas por los productos ofrecidos por otras empresas, adaptándose con flexibilidad a las necesidades de sus clientes. Las Pymes investigadas tienen la capacidad de proporcionar elementos de valor en sus productos, bienes y servicios, sumados a los eventos de calidad en ISO y mejora continua que realizan para proporcionar un mejor servicio. Con lo anterior, la Pyme aprende y se desarrolla promovida por sus clientes y proveedores en nuevos procesos para las mejores prácticas en su manufactura. Se encuentran algunas evidencias en el uso que hace el emprendedor de las tecnologias de la información (TIC), principalmente del software, como una plataforma administrativa de todos los procedimientos de entrada-proceso-salida de la Pyme, por ejemplo, el Enginneering Ressource Planning (ERP), el Balance Score Card y el Solomon IV.

\section{Control de la información por el emprendedor}

Producto del análisis de la investigación en México, encontramos propiedades relacionadas con la intensa actividad del emprendedor para allegarse y establecer formas de controlar la información del exterior y del interior de su empresa. Así, identificamos evidencias de un tipo de señales fuertes y otro tipo de señales débiles para el control de la información por el emprendedor. 
Las Pymes estudiadas obtienen beneficios por el control de la información en señales fuertes de manera paralela al servicio que otorgan a sus clientes. Las Pymes encuentran nuevos procesos y procedimientos de fabricación migrando a otras tecnologías que las hagan más eficientes para satisfacer las necesidades de sus clientes, evolucionando como proveedores confiables y sólidos, que incluso en varios casos de las Pymes investigadas han sido recomendadas como proveedores por sus actuales clientes con otras empresas del grupo o filiales, lo que ha redundado en el fuerte crecimiento de las mismas. También se han beneficiado con sus proveedores en el servicio de capacitación y en captar información para atacar nuevos mercados.

Con respecto a la propiedad del tipo de información vigilada existen tres dimensiones identificadas en los relatos de las entrevistas que son complementarias, como la información de oportunidades nuevas en su mercado que vigila el emprendedor, la información de nuevos nichos de mercado y la información de incertidumbre de mercado, las cuales se fusionaron y constituyeron una dimensión que denominaremos estrategias de mercado. Esto en virtud de que el emprendedor utiliza la información de su mercado para iniciar acciones que favorezcan a su Pyme, y sobre las oportunidades que le ofrece su mercado, en especial con clientes nacionales y extranjeros. Posee información del tipo de un "sistema de alerta temprano" de las condiciones favorables o desfavorables de sus diferentes mercados y los probables escenarios para incrementos, estabilidad, decrementos o cancelación de sus ventas; de ahî que, en consecuencia, en los dos últimos escenarios aproveche las oportunidades para abrir un nuevo nicho de mercado e introducir los mismos o nuevos productos, bienes o servicios.

Entonces podemos decir que la competitividad en las Pymes no proviene solamente de la difusión de las nuevas tecnologías de gestión y de productos, que en muchos casos no sólo dependen de la inversión material, sino también de nuevas formas complejas de gestión y de organización, y de este modo de una inversión inmaterial en investigación y desarrollo, y en una vigilancia importante de la información. La inversión inmaterial permite a las Pymes, a largo plazo, la renovación sistemática de sus productos y de la organización de su producción; implica, pues, innovación en todos los niveles, y ésta implica tener en cuenta el ambiente ("la escucha del ambiente") y, por consiguiente, no sólo la presencia de una vigilancia tecnológica, competitiva y comercial implícita, sino relativamente organizada. Esta información es costosa, más aun cuando cambia rápidamente. Las Pymes son particularmente vulnerables en este sentido, a causa de sus recursos limitados para crear, obtener y evaluar esta información. Por esta razón deben asociarse o construir redes que les permitan obtener la información necesaria a un costo menor. Como sus recursos son limitados, estas redes les permiten disminuir los costos operativos para obtener la información, ya sea tecnológica, comercial o del mercado.

En las Pymes, la mayoría de las veces las redes son informales (amigos, contactos sociales, profesionales, etcétera), y se vuelven más estructuradas y formalizadas (programas de ayuda, consultorías, Centros de investigación, instituciones de educación superior, etcétera) a medida que la empresa se desarrolla y estructura su vigilancia. Estas redes permiten a las Pymes generar más dinámicas para acondicionar su ambiente, a fin de reducir la incertidumbre y administrar mejor el largo plazo.

Otra dimensión del control de la información de señales débiles es la asistencia a ferias y exposiciones industriales, la cual se mantuvo presente en la mayoría de los casos. El emprendedor la utiliza como fuente importante de información para la inversión y el desarrollo de maquinaria y tecnología de punta, como oportunidades o nuevos nichos de mercado como parte de su estrategia de crecimiento dentro de una innovación en pequeños pasos y en forma de torbellino.

\section{Perspectivas de las competencias esenciales de las Pymes}

Otra categoría que incluimos en el esquema conceptual de la figura 3 es el de las competencias esenciales, el cual según Prahalad \& Hamel (1990) evolucionan del producto clave al de competencia esencial. Ellos estudiaron el comportamiento estratégico de empresas japonesas y de Occidente para determinar lo que caracterizó el éxito en común y a largo plazo de esas compañías, y encontraron en su investigación lo que ellos denominaron core competencies (cc), que por su traducción se refiere a competencias esenciales, definidas de la siguiente manera:

\footnotetext{
...las cualidades intrinsecas del grupo empresarial que lo impulsa al éxito económico; estas cualidades se expresan a través del aprendizaje colectivo en la organización, específicamente la coordinación diversa de habilidades de producción y la integración de múltiples corrientes de tecnología en las empresas del grupo y en los productos finales (Prahalad \& Hamel, 1990).
} 
Además, agregan que las competencias esenciales son la comunicación, la participación y un profundo compromiso a trabajar a través de los limites de la organización; las competencias esenciales no disminuyen con el uso, mientras que el conocimiento sí se debilita por no usarse; las competencias son el pegamento que une la existencia del negocio.

Leonard-Barton (1992) define las capacidades esenciales, manteniendo la misma connotación de competencia esencial o clave:

...como un sistema de conocimientos interdependientes que provee una ventaja competitiva y que posee cuatro dimensiones: conocimientos y habilidades de los empleados; sistemas técnicos, que consisten en acumular, codificar y estructurar conocimiento tácito; sistemas de dirección que incluyen procedimientos formales e informales de crear y controlar el conocimiento, como son los programas de aprendizaje, redes de socios y sistemas de incentivos; y finalmente normas y valores asociados con varios tipos de conocimientos y con el proceso de creación y control de conocimientos (Leonard-Barton, 1992).

Por otra parte, también Levy-Leboyer (1996) establece que las competencias individuales y competencias clave de la empresa están evidentemente en estrecha relación; las competencias de la empresa están constituidas, ante todo, por la integración y la coordinación de las competencias individuales, al igual que, a otra escala, las competencias individuales representan una integración y una coordinación de savoir-faire, conocimientos y cualidades individuales. De ahí la importancia para la empresa de administrar bien su stock de competencias individuales, tanto actuales como potenciales. Lo anterior se refuerza con la propuesta que realizaron años atrás Prahalad \& Hamel (1990):

En los años noventa [...] los mandos dirigentes juzgaban por sus aptitudes para identificar, cultivar y explotar las competencias claves que hacen posible el crecimiento [...] competencias claves que están constituidas por la experiencia colectiva de la empresa y que concierne especialmente a la coordinación de diversas capacidades de producción y de la integración de líneas de tecnología (Prahalad \& Hamel, 1990).

Prahalad \& Hamel (1990), derivado de sus largos estudios, proponen dentro de sus postulados para alcanzar lo que nosotros llamamos el fuerte crecimiento de la empresa, diseñar una arquitectura estratégica, la cual consiste en un mapa o camino que señale qué competencias esenciales se deben construir y qué tecnologías las constituyen, contemplando a las personas y los proyectos relacionados con las competencias esenciales. Estos autores señalaron que las organizaciones supervivientes de la primera etapa de la competencia global convergian en estándares similares de calidad y costos de los productos, factores que serían cada vez menos importantes como fuente de ventaja competitiva. Por tanto, en el largo plazo, el desempeño superior derivaba de la habilidad de la dirección para consolidar tecnologias y habilidades de producción en competencias que hicieran más poderosa a cada empresa del grupo empresarial y que la ayudaran a adaptarse a las oportunidades cambiantes del medio.

Prahalad \& Hamel (1990) establecen:

...resulta paradójico constatar que los altos mandos dirigentes dediquen tanto esfuerzo a los problemas planteados por el reparto de los recursos financieros y no lleven a cabo un proceso comparable que afecte a las cualidades individuales que constituyen las competencias clave de la empresa (Prahalad \& Hamel, 1990).

Para que la empresa tenga un fuerte crecimiento requiere contar con una arquitectura estratégica de sus competencias esenciales o lo que nosotros precisamos como la combinación de recursos que distinguen a la empresa. Esta combinación de recursos se convierte en una administración estratégica que se construye bajo una visión y proyección en el tiempo futuro de la empresa, esto es, una visión y un pensamiento a largo plazo, con el fin de anticiparse a los cambios y liberar las resistencias al mismo, y que es requerida como una condición para el éxito de la empresa. Los altos directivos deben tener una opinión sobre cuáles son los nuevos beneficios que se ofrecerán a los clientes en la próxima década, sobre cuáles serán las nuevas competencias esenciales que se necesitarán para ofrecer esos beneficios y sobre cómo habrá de modificarse la relación con los clientes para que éstos puedan acceder más eficazmente a esos beneficios. Las competencias esenciales, en complemento con la dinámica del emprendedor, explican el fuerte crecimiento del grupo de las Pymes manufactureras estudiadas, ya que los emprendedores dentro de su dinámica tenían identificadas cuáles eran sus competencias esenciales en ese momento y cuáles las nuevas competencias esenciales 
a construir, denominadas arquitectura estratégica, para enfrentar los retos de los nuevos mercados; sabían si las competencias actuales estaban erosionándose lentamente o estaban reforzándose, eran capaces de distinguir entre los negocios a aquéllos que debían deshacerse o las competencias que debian conservar; sabian qué competencias estaban adquiriendo otras empresas y reconocían que los competidores a los que se enfrentaban en el terreno de las competencias esenciales podian no ser los mismos que participaban en el terreno de los productos finales (Prahalad \& Hamel, 1990).

De manera informal, es una constante la capacitación sobre la acción de las competencias adquiridas de los procedimientos de fabricación eficientes, ya que habilitan instructores internos con el personal clave durante el proceso de producción o también realizan entrenamientos cruzados y roles de puestos. La competitividad de las Pymes con fuerte crecimiento estudiadas en la región de San Luis Potosí (Molina, 2014) es debido a la forma en que el emprendedor administra sus recursos de competencias esenciales, la forma de combinarlos con las competencias laborales de su personal, aunado con el buen estado de operación de los equipos, maquinaria y tecnología. Esto hace que logren procesos y procedimientos de fabricación con la capacidad y calidad de una Pyme proveedora de clase mundial. Todo esto certificado en sus procesos con los sistemas ISO, en la mayoría de los casos de las Pymes investigadas.

Por otra parte, también las competencias esenciales - cuando se genera una sinergia y un valor agregado particular para el mercado- (Torkkeli \& Tuomine, 2002, citados por Julien, 2006) crean una combinación virtuosa, y este portafolio de competencias, como tarea colectiva, comprende también los vínculos personales y de fidelidad con los proveedores y los distribuidores, como con los clientes u otras empresas, ventajas aún más dificiles de imitar porque cuentan con varias producciones e intercambios complejos de saberes y saberhacer (Dyer \& Singh, 1998). Estos vínculos permiten una producción y una distribución que mantienen la distinción. Sin embargo, esta combinación no es óptima, en general, porque no hay una buena manera de ser, según su mercado y su industria.

Los emprendedores de Pymes con fuerte crecimiento tienen claro qué competencias esenciales construir en el futuro para hacer frente a los nuevos desafios y consolidación de su crecimiento en el mercado, producto de la implementación de estrategias hacia den- tro de su Pyme que le permiten llegar a ellas con el soporte de su personal, y de estrategias hacia fuera de su empresa haciendo uso de su red de negocios. Con la visión de crecimiento, resultado de su plan estratégico, optimiza sus recursos y se proyecta al futuro para consolidar la integración de las Pymes del sector, uniendo sus competencias esenciales e integrando una red de subcontratación sólida con las empresas grandes del sector automotriz, electrodoméstico y aeroespacial.

En resumen, la arquitectura estratégica tiene que ver con la administración estratégica de las competencias esenciales de la Pyme manufacturera, y está estrechamente relacionada con la percepción de la innovación, con el control de la información y con el capital social del emprendedor.

\section{Aportaciones de las investigaciones nacionales e internacionales sobre el emprendimiento y crecimiento de las Pymes}

El común denominador de los estudios internacionales sobre el fuerte crecimiento de las Pymes Las Gacelas es que están basados en análisis cuantitativos y probabilísticos, pero también cualitativos, lo que implica una muestra representativa de las poblaciones y, en algunos casos, se efectúa con la población total, como en el caso de Suiza, lo que implica también desplegar una gran cantidad de recursos económicos para la investigación y que sean sustentandas por los ministros de economía y/o universidades públicas o privadas, como en el caso de Woywode \& Lessat (2001) en Alemania; Calvo \& Lorenzo (2001) en España; Mustar (2001) en Francia; Davidsson \& Delmar (2001) en Suiza, Kemp, Verhoeven \& Kreijen (2001) en los Países Bajos; Zaralis (2001) en Grecia; Bramanti (2001) en Italia, y Julien (2001) en Quebec. Los datos que obtuvieron son relevantes para el conocimiento y comportamiento económico-social de las Pymes en esos países, y generalizable para países con el mismo desarrollo socioeconómico e industrial, por lo que no podemos generalizar a México. Es por ello que realizamos el estudio entre 2006 y 2007 en la región de San Luis Potosí, y en este último apartado presentamos algunos resultados y su comparación con los estudios internacionales.

En el caso de los estudio internacionales abarcaron periodos de observación y análisis en algunos casos de hasta diez años anteriores a la fecha de su publicación; 
por ejemplo, encontraron que el 10\% de las empresas investigadas pertenecian a las Pymes de nueva creación, con gran crecimiento y expansión económica, por encima de las empresas grandes y consolidadas. Tienen datos de que la forma jurídica de su integración influye en su crecimiento y también en las prácticas de investigación y desarrollo; además, la exportación y características individuales como la edad, experiencia y formación del empresario son variables para el crecimiento.

Encontraron que el tamaño y edad de la empresa son factores para el crecimiento, y que el capital intelectual representa ventajas competitivas para la Pyme, así como la inversión en nueva tecnología y la innovación. Se enfrentaron a problemas dificiles de delimitar dentro del sondeo escrito que realizaron como las ideas del fundador, las estructuras formales e informales de la organización y la estrategia de la empresa, determinantes en gran parte del éxito de la empresa; expresan también que las cualidades psico-sociológicas del empresario no exploradas (como sus ambiciones, su audacia y sus aspiraciones de beneficios) son factores explicativos importantes para el crecimiento rápido de las empresas.

En España, los resultados sobre las empresas de fuerte crecimiento fueron: las empresas son jóvenes y pequeñas poseen tecnología media y alta, con un producto diferenciado, venden sus productos a mercados amplios, en expansión, y con orientación internacional; innovan, utilizan los últimos desarrollos tecnológicos y realizan actividades de investigación y desarrollo tanto directas como complementarias; 40\% de Pymes de fuerte crecimiento (EFC) pertenecen a un grupo industrial. La introducción de innovación de procesos es igualmente un hecho diferencial de las EFC, lo mismo que la utilización de nuevas tecnologías dentro de los procesos de producción: más de $80 \%$ sacan provecho de los más recientes descubrimientos tecnológicos. Las EFC invierten más en actividades de I+D, venden sus productos en grandes mercados y tienen una expansión dirigida a mercados internacionales.

Los estudios de Francia ponian su acento en varios elementos comunes que desempeñan un papel relevante en el proceso de fuerte crecimiento, éstos son: la cooperación de estas empresas con actores diferentes para innovar, la particular gestión de la organización que constituye una empresa de fuerte crecimiento, y el financiamiento y el papel de las políticas públicas. Esta investigación critica los estudios internacionales que pretenden ser una receta que describa un solo tipo de crecimiento de las Pymes, argumentando que el fuerte crecimiento es un proceso y que no hay una única manera de hacerlo que sea la mejor, sino que, más bien, existen diferentes configuraciones que contrastan el fuerte crecimiento de las Pymes.

El estudio de Suecia exploraba la contribución de las empresas de alto crecimiento a la creación de empleo en ese país. Utilizaron la población completa de las empresas existentes desde 1996 con más de 20 empleados, analizando su desarrollo anual durante diez años. Los resultados indicaron que las empresas jóvenes y medianas creaban la mayor parte de los empleos.

El estudio en los Países Bajos encontró que la mitad del empleo bruto creado por las empresas existentes corresponde a empresas en crecimiento rápido. Las EFC suelen reducir su crecimiento al cabo de un tiempo. Las empresas jóvenes aportan una gran contribución al incremento de las empresas en crecimiento rápido. El Gobierno holandés crea las condiciones para una competencia dinámica y evita las alianzas que limitan la competencia, además trata de eliminar los obstáculos a los que se enfrentan lo empresarios, como las debilidades del mercado, y estimula la creación de empresas nuevas. El Gobierno ha tomado medidas adicionales para las EFC, como la extensión del alcance de las redes, el fomento de la formación de los empresarios para que adquieran las aptitudes necesarias y la mejora de la información.

El estudio en Grecia se centró en la influencia de las Pymes con fuerte crecimiento sobre la creación de empleo en el sector manufacturero griego. Los resultados indicaron que el tamaño de las empresas, los gastos en innovación, las exportaciones, la rentabilidad, la ubicación y el crecimiento del sector tienen una influencia sobre la posibilidad de pertenecer a los grupos de Pymes con fuerte crecimiento.

El estudio que utilizó un método cuantitativo y cualitativo fue la investigación de Julien (2001) sobre las Pymes en fuerte crecimiento, en Quebec, Canadá. Podemos observar que conociendo los resultados de los estudios internacionales y en especial este último de Quebec, la investigación en México se enriquece debido a que introduce la exploración de la dinámica del emprendedor y las competencias esenciales actuales y por construir, como aspectos importantes en el fuerte crecimiento del grupo de Pymes estudiadas. 
En la tabla 1 podemos observar la comparación de los sistemas de organización que implementan las Pymes para su fuerte crecimiento, destacando en los estudios de Quebec el énfasis en la forma particular en que éstas se organizan; es decir, es uno de los dos temas más importantes y de interés que explora y aporta el grupo de Canadá. En México importa por parte del emprendedor tener una infraestructura y un estricto control de costos para ofrecer un servicio acorde con las necesidades de sus clientes.

En los resultados de las investigacionesnacionales e internacionales sobre el comportamiento del empresario para el fuerte crecimiento de las Pymes se observa la poca exploración de los estudios internacionales de la categoría en comparación con el estudio en México. Conocer el impacto que tiene el emprendedor en la dinámica que le imprime en la conducción de su empresa para el crecimiento fue un hallazgo, ya que el emprendedor pondera los valores sociales en la relación con empleados, clientes y proveedores, da importancia al "saber ser", continuamente cambia de perspectiva "del hacer las cosas" por la innovación, involucra y valora a sus empleados en sus tareas, agrega elementos de valor para el cliente, despliega versatilidad en la conducción de su empresa, busca la oportunidad para cultivar relaciones humanas, apasiona y arraiga a sus clientes e incluso tiene reingreso de clientes, mantiene un alto empuje a pesar de los escasos apoyos del Gobierno e instituciones destinadas a apoyarlas y de las grandes crisis económicas en México en las últimas décadas.
Por otra parte, las tres investigaciones tienen hallazgos interesantes con respecto a la perspectiva de los recursos y capacidades de la Pyme; sin embargo, la investigación en México demostró que las Pymes combinan los recursos atendiendo a sus competencias esenciales, especificando las actuales y las que se requieren desarrollar para lograr el fuerte crecimiento, esto es, lo que conocemos como arquitectura estratégica. En la tabla 2 podemos observar que en el estudio internacional se destaca la I+D para apuntalar el crecimiento de las Pymes (aunque en Quebec la capacitación y actualización del personal estratégico es clave, así como los procesos de mejora continua, para lograr las certificaciones en calidad), y en México queda evidencia de que llevar a las Pymes a una especialización del saber-hacer, las formas de comercialización y la competencia para el uso de procesos rápidos y flexibles en su producción, son básicos para su crecimiento.

Por otra parte, también los tres estudios ponen atención en las evidencias que tienen que ver con la estrategia que implementan las Pymes para lograr el fuerte crecimiento (tabla 3); sin embargo, en los estudios internacionales la cultura de cooperación y asociación entre empresas está más arraigado, mientras en la presente investigación solamente se detectó que existen proyectos importantes para hacer frente a las demandas de subcontratación de las grandes empresas y la competencia de Pymes del Oriente.

Tabla 1.

Resultados de las investigaciones sobre los sistemas de organización de las Pymes para el fuerte crecimiento.

\begin{tabular}{|c|c|c|}
\hline Estudios de la OCDE & Estudios de Quebec & Investigación de México \\
\hline $\begin{array}{l}\text { La forma juridica de su integración influye } \\
\text { en su crecimiento. } \\
\text { Las empresas jóvenes y medianas crean la } \\
\text { mayor parte de los empleos. }\end{array}$ & $\begin{array}{l}\text { Organización particularmente compleja, } \\
\text { con la aportación de recursos exteriores } \\
\text { para completar cuando sea necesaria. } \\
\text { Nómina con ejecutivos de diversas } \\
\text { profesiones. } \\
\text { Prácticas organizacionales } \\
\text { descentralizadas, es decir, los empleados } \\
\text { participan en las decisiones de compra de } \\
\text { equipos y en la estrategia de innovación. } \\
\text { Motivación con participación de utilidades. } \\
\text { Sus objetivos se centran en utilidades, } \\
\text { ambiente agradable, retos de crecimiento } \\
\text { y retos de trabajo en equipo. } \\
\text { La producción en algunos casos es } \\
\text { sobremedida. }\end{array}$ & $\begin{array}{l}\text { Capacidad y calidad instalada del sistema } \\
\text { de organización y de producción actual. } \\
\text { Control administrativo estricto en costos y } \\
\text { reinversión de capital. }\end{array}$ \\
\hline
\end{tabular}

Fuente: Elaboración propia. 
Tabla 2.

Resultados de las investigaciones sobre los recursos y capacidades de las Pymes para el fuerte crecimiento.

\begin{tabular}{|c|c|c|}
\hline Estudios de la OCDE & Estudios de Quebec & Investigación de México \\
\hline $\begin{array}{l}\text { La introducción de innovación de procesos } \\
\text { y la utilización de nuevas tecnologias } \\
\text { dentro de los procesos de producción. } \\
\text { Los resultados indicaron que el tamaño } \\
\text { de las empresas, las exportaciones, la } \\
\text { rentabilidad, la ubicación y el crecimiento } \\
\text { del sector influyen sobre la posibilidad } \\
\text { de pertenecer a los grupos de Pymes con } \\
\text { fuerte crecimiento. }\end{array}$ & $\begin{array}{l}\text { Tecnologias materiales estaban } \\
\text { actualizadas. } \\
\text { Certificadas con ISO o más (por ejemplo } 6 \text { o). } \\
\text { La formación continua es muy importante, } \\
\text { con } 5 \% \text { de asignación de la nómina. }\end{array}$ & $\begin{array}{l}\text { Desarrollo y especialización de nuevas } \\
\text { competencias esenciales para fabricar } \\
\text { nuevos productos, bienes y servicios. } \\
\text { Capacidad de comercialización y } \\
\text { negociación de sus productos, bienes y } \\
\text { servicios. } \\
\text { Competencias laborales del PD, su perso- } \\
\text { nal clave y de nuevo ingreso en el "oficio" } \\
\text { de la empresa. } \\
\text { Competencias en el uso de equipos } \\
\text { y tecnologia para procesos rápidos y } \\
\text { flexibles. } \\
\text { Organización que aprovecha los recursos } \\
\text { que le proporciona la transición como } \\
\text { Pyme familiar. }\end{array}$ \\
\hline
\end{tabular}

Fuente: Elaboración propia.

Tabla 3.

Diferencias y aportaciones en los resultados de las investigaciones sobre las estrategias que implementa las Pymes para el fuerte crecimiento.

\begin{tabular}{|c|c|c|}
\hline Estudios de la OCDE & Estudios de Quebec & Investigación de México \\
\hline $\begin{array}{l}\text { Las Pymes de nueva creación tienen } \\
\text { gran crecimiento y expansión económica, } \\
\text { por encima de las empresas grandes y } \\
\text { consolidadas. } \\
\text { El fuerte crecimiento se debe a las } \\
\text { prácticas de investigación y desarrollo, a la } \\
\text { exportación y a características individuales } \\
\text { como la edad joven de la Pyme. } \\
\text { Pymes con un producto diferenciado, } \\
\text { venden sus productos a mercados } \\
\text { amplios, en expansión y con orientación } \\
\text { internacional. } \\
\text { El } 40 \% \text { de las Pymes de fuerte crecimiento } \\
\text { pertenecen a un grupo industrial. } \\
\text { Más de } 80 \% \text { saca provecho de los más } \\
\text { recientes descubrimientos tecnológicos. } \\
\text { La cooperación de estas empresas con } \\
\text { actores diferentes para innovar, el } \\
\text { financiamiento y el papel de las políticas } \\
\text { públicas. }\end{array}$ & $\begin{array}{l}\text { Decisiones estratégicas compartidas. } \\
\text { Un comité de gestión analizaba, elaboraba, } \\
\text { revisaba y actualizaba el plan estratégico. } \\
\text { Acceso a información y participación en } \\
\text { acciones. } \\
\text { El liderazgo se basa en una mezcla entre } \\
\text { formación y experiencia. } \\
\text { Alta formación del nivel medio superior } \\
\text { para arriba de la dirección y empleados. } \\
\text { Los factores de éxito en el mercado se } \\
\text { deben a la fuerte relación con sus clientes } \\
\text { y a la alta calidad en el servicio. } \\
\text { Su orientación al mercado, por contactos } \\
\text { permanentes y directos con su cliente. }\end{array}$ & $\begin{array}{l}\text { PD aplica criterios para la selección de } \\
\text { clientes. } \\
\text { Organización que busca la diversificación } \\
\text { en productos, bienes y servicios. } \\
\text { Organización crítica y exigente con los } \\
\text { servicios externos de consultoría. } \\
\text { Organización que busca integrarse } \\
\text { estratégicamente con otras Pymes. } \\
\text { Los proveedores le agregan elementos de } \\
\text { valor a los servicios a la Pyme. }\end{array}$ \\
\hline
\end{tabular}

Fuente: Elaboración propia.

\section{CONCLUSIONES}

Como lo plantean Strauss \& Corbin (1998) nos aproximamos a la construcción de un esquema explicativo sistemático del perfil psicosociológico, que integra en él los conceptos y las relaciones, como se muestra en la tabla 1, la cual sirve de referencia para la discusión y evolución de la teoría del emprendimiento que deseamos proponer a la comunidad académica y científica de las Ciencias Administrativas y a los emprendedores visionarios que focalizamos desde el inicio de esta obra. Con el esquema conceptual se describen las características de un grupo de Pymes manufactureras con fuerte crecimiento en la región y, en seguida, 
enunciamos los hallazgos que encontramos en la investigación sobre las dimensiones endógenas y exógenas en las Pymes que explican las características de su fuerte crecimiento y que se destacan por sus vínculos en red con diferentes propiedades.

Por último, el estudio internacional de la OCDE pone énfasis en la importancia del fuerte crecimiento de las Pymes debido a las políticas económicas implementadas por los gobiernos de los países donde se desarrollaron las investigaciones. En la investigación internacional se detectó que el Gobierno trata de eliminar los obstáculos a los que se enfrentan los empresarios, como las debilidades del mercado. Asimismo, estimula la creación de empresas nuevas, además de que el Gobierno tomó medidas adicionales para las Pymes con fuerte crecimiento como la extensión del alcance de las redes y el fomento de la formación de los empresarios para que adquieran las aptitudes necesarias y la mejora de la información. En la investigación de Quebec, el Gobierno genera de manera permanente recursos económicos y asesorías para las Pymes. En el caso de las Pymes investigadas en el presente estudio, el apoyo del Gobierno ha sido escaso, y sólo el Gobierno estatal generó un programa de desarrollo de proveedores para Pymes manufactureras subcontratistas de grandes compañias del sector de electrodomésticos y automotriz en el periodo de 2005 a 2007. A nivel nacional, en el 2013 el Gobierno federal, a través de el Instituto Nacional del Emprendedor (Inadem), apoya a pequeñas empresas con base tecnológica en su programa denominado Red nacional del emprendedor y con las convocatorias de financiamiento que cerraron en julio de 2013, y que están siendo evaluadas si son eficientes y efectivas para apoyar a las Mipymes en México.

\section{REFERENCIAS}

Álvarez, M. (2003). Competencias centrales y ventaja competitiva: el concepto, su evolución y su aplicabilidad. Revista Contaduría y Administración, (209), 5-22.

Barney, J. (1986). Strategic Factors Markets: Expectations, Lucks, and Business Strategy. Management Science, 32(10), 1231-1241.

Barney, J. (1991). Firm Resources and Sustained Competitive Advantage. Journal of Management, 17(1), 99-120.

Barney, J. B. (1996). The Resource-Based Theory of the Firm. Organization Science: A Journal of the Institute of Management Sciences, 7(5), 469-469.

Bramanti, A. (2001). Les PME à forte croissance en Italie: succès étonnants et désagréables surprises. Revue Internationale PME, 14(3-4), 92-127.
Bruyat, C. \& Julien, P. A. (2001). Defining the field of research in entrepreneurship. Journal of Business Venturing, 16(2), 165.

Calvo, J. L. \& Lorenzo, M. J. (2001). Une caractérisation des entreprises manufacturières espagnoles à forte croissance: 1994-1998. Revue Internationale PME, 14(3-4), 45-66.

Conner, K. R. \& Prahalad, C. K. (1996). A Resource-Based Theory of the Firm: Knowledge versus Opportunism. Organization Science: A Journal of the Institute of Management Sciences, 7(5), 477-501.

Davidsson, P. \& Delmar, F. (2001). Les entreprises à forte croissance et leur contribution à l'emploi: les cas de la Suède 1987-1996. Revue Internationale PME, 14(3-4), 164-187.

Dyer, J. H. \& Singh, H. (1998). The Relational View: Cooperative Strategy and Sources of Interorganizational Competitive Advantage. Academy of Management Review, 23(4), 660-679.

García, E. (1991). Las características personales del empresario en la creación de empresa (Tesis de doctorado). Universidad de Pamplona: España.

George, C. (2005). Historia del pensamiento administrativo (2da. ed.). México: Pearson Prentice Hall.

Glaser, B. G. \& Strauss, A. L. (1967). The Discovery of Grounded Theory: Strategies for Qualitative Research. Chicago: Aldine.

González, F. (2006). Tecnología de información administrativa en culturas organizacionales innovadoras. Factores cualitativos de autonomía y control (Tesis de doctorado). Universidad Autónoma de Aguascalientes: México.

Grant, R. M. (1991). The Resource-Based Theory of Competitive Advantage: Implications for Strategy Formulation. California Management Review, 33(3), 114.

Hamel, G. \& Prahalad, C. K. (1995). Compitiendo por el futuro: estrategia crucial para crear los mercados del mañana (2a. reimpresión, marzo 1999). España: Ariel Sociedad Económica.

Instituto Nacionla de Estadística y Geografía (INEGI) (2009). Censos Económicos. México: INEGI.

Julien, P. A. (2001). Les PME à forte croissance et la métaphore du jazz. Comment gérer l'improvisation de façon cohérente. Revue Internationale PME, 14(3-4), 129-161.

Julien, P. A. (2006). Réseautage et innovation: le cas des PME manufacturières d'une région en reconversion. Économie et société (série W 'Dynamique technologique et organisation'), 9(1), 75-100.

Julien, P. A. \& Marchesnay, M. (1997). L'entrepreneuriat. Paris: Ed. Económica.

Julien, P. A. \& Molina, R. (2012). Una teoría sobre el emprendimiento regional en la economía del conocimiento: una metáfora de las novelas policiacas. México: PEARSON.

Kemp, R., Verhoeven, W. \& Kreijen, M. (2001). La croissance et la décroissance des entreprises aux Pays-Bas. Revue Internationale PME, 14(3-4), 189-210.

Leonard-Barton, D. (1992). Core Capabilities and Core Rigidities: A Paradox in Managing New Product Development. Strategic Management Journal, 13(5), 111-125. 
Levy-Leboyer, C. (1996). La gestion des compétences, les éditions d'organisation. Paris, Francia: Ediciones Gestión.

Miles, R. E. \& Snow, C. C. (1984). Designing Strategic Human Resources Systems. Organizational Dynamics, 13(1), 36-52.

Molina, R. (2014). El fuerte crecimiento de la Pyme desde la perspectiva de las competencias esenciales: la empresa Gacela. México: Universidad de Ganajuato.

Mustar, P. (2001). Diversité et unité des entreprises à forte croissance du secteur manufacturier en France. Revue Internationale PME, 14(3-4), 68-89.

Organización para la Cooperación y el Desarrollo Económicos (OCDE) (1998). Principaux indicateurs de la science et de la technologie, Paris.

Penrose, E. T. (1952). Biological Analogies in the Theory of the Firm. American Economic Review 42(5), 804.

Penrose, E. T. (1959). The Theory of the Growth of the firm. Gran Bretaña: Oxford University Press.

Porter, M. E. (1982). Estrategia competitiva: Técnicas para el análisis de los sectores industriales y de la competencia. México, DF: CECSA.

Prahalad, C. K. \& Hamel, G. (1990). The Core Competence of the Corporation Harvard Business Review, 41(3), 79-93.

Rodríguez, J. (2002). Administración de pequeñas y medianas empresas. México Cengage Learning Editores.
Rumelt, R. P. (1991). How Much Does Industry Matter? Strategic Manage Journal, 12(3), 167-185.

Strauss, A. \& Corbin, J. (1998). Basics of Qualitative Research: Techniques and Procedures for Developing Grounded Theory. Thousand Oaks, London, New Delhi: Sage.

Suárez-Núñez, T. (2002). La estrategia, la tecnología y la competitividad en la pequeña empresa industrial mexicana. Un estudio multicaso (Tesis de doctorado). Universidad Autónoma Metropolitana: México.

Wernerfelt, B. (1984). A Resource-Based Theory of the Firm. Strategic Management Journal, 5(1), 99-120.

Woywode, M. \& Lessat, V. (2001). Les facteurs de succès des entreprises á croissance rapide en Allemagne. Revue Internationale PME, 14(3-4), 17-43.

Yeung, A., Ulrich, D., Nason, S., Von, G. \& Mary, A. (2000). Las capacidades de aprendizaje en la organización. Cómo aprender a generar y difundir ideas con impacto. México: Oxford University Press.

Zaralis, G. (2001). Petit et moyennes entreprises à forte croissance et emploi dans le secteur manufacturier grec. Revue Internationale PME, 14(3-4), 212-239.

Zimmerer, T. W. \& Scarborough, N. M. (1998). Essentials of entrepreneurship and small business management (2nd Ed.). New York: Prentice Hall. 